Revista de Metalurgia

Abril-Junio 2019, 55 (2) e142

ISSN-L: 0034-8570

https://doi.org/10.3989/revmetalm.142

\title{
Estudio de la extracción de tierras raras ligeras a partir de la extracción líquido - líquido utilizando ácidos organofosforados y ácido ascórbico
}

\author{
Gabriel Santos ${ }^{\mathrm{a}}$, Ysrael M. Vera ${ }^{\mathrm{a}, \bigotimes}$ \\ ${ }^{a}$ Centro de Tecnologia Mineral, Av. Pedro Calmon 900, Ciudad Universitaria, Rio de Janeiro, CEP 21941-908, Brasil \\ (®Autor para la correspondencia: yvera@cetem.gov.br)
}

Enviado: 4 Junio 2018; Aceptado: 13 Febrero 2019; Publicado on-Line: 19 Junio 2019

\begin{abstract}
RESUMEN: La extracción líquido - líquido (ELL) o extracción por Solvente (SX) es la técnica más utilizada, actualmente, para promover la separación de los Elementos Tierras Raras (ETRs), siendo los agentes de extracción ácidos organofosforados los más empleados. El uso de estos reactivos de extracción parcialmente saponificados aumenta tanto la extracción como la selectividad de la extracción de los ETRs. Sin embargo, esta práctica genera efluentes líquidos conteniendo sodio, amonio, magnesio o calcio que si no se eliminan pueden ocasionar daños al medio ambiente. En los últimos años se han buscado alternativas a la práctica de saponificación. La utilización de agentes acomplejantes, como el ácido ascórbico, puede ayudar a realizar la separación de los ETRs más eficientemente y sin provocar daños al medio ambiente por ser una sustancia biodegradable. El presente trabajo tuvo como objetivo evaluar la influencia de la concentración de ácido ascórbico sobre la extracción de lantano y didimio $(\mathrm{Pr}+\mathrm{Nd})$ a partir de la ELL. Se utilizaron tres agentes de extracción ácidos organofosforados (D2EHPA, P507 y Cyanex272). Se verificó que, con el aumento de la concentración del ácido ascórbico, hubo un incremento en el porcentaje de extracción y en la selectividad de la extracción de los ETR. Se definió, además, que el agente de extracción más eficiente en las condiciones estudiadas fue el P507. Consideramos que las mejores condiciones para realizar la separación entre La/didimio fue obtenida con el agente de extracción P507 y concentración de ácido ascórbico $0,5 \mathrm{~mol} \cdot \mathrm{L}^{-1}$. En esta condición se obtuvo una extracción de La, Pr y $\mathrm{Nd}$ igual a $12,3 \pm 1,3 \%, 56,4 \pm 1,0 \%$ y $64,8 \pm 1,7 \%$, respectivamente, así como un factor de separación de $9,3 \pm$ 0,8 y didímio en fase orgánica con $81,4 \pm 0,5 \%$ de pureza.
\end{abstract}

PALABRAS CLAVE: Ácido ascórbico; Cyanex272; D2EHPA; Elementos tierras raras; Extracción Líquido-Líquido; P507

Citar como/Citation: Santos, G.; Vera, Y.M. (2019). "Estudio de la extracción de tierras raras ligeras a partir de la extracción líquido - líquido utilizando ácidos organofosforados y ácido ascórbico". Rev. Metal. 55(2): e142. https:/l doi.org/10.3989/revmetalm.142

\begin{abstract}
Study on extraction of light rare earth from liquid - liquid extraction using organophosphoric acids extractants and ascorbic acid. The Liquid - liquid extraction (LLE) or solvent extraction (SX) is the most widely technique used to separate the Rare Earth Elements (REEs). The saponification of the extractants has been used as an efficient practice to promote this separation, however this process produces wastewater containing ammonia nitrogen, sodium, magnesium or calcium ions that must be removed from solution otherwise could cause environmental pollution. There is a concern about separating efficiently rare earth elements by alternative methods to saponification. The use of complexing agents, such as ascorbic acid, may be used as an alternative to maintain the efficiency of the separation process and without causing any harm to the environment because it is a biodegradable substance. This study aimed to evaluate the influence of ascorbic acid concentration over lanthanum and didymium $(\mathrm{Pr}+\mathrm{Nd})$ extraction by liquid - liquid extraction. Three acids organophosphorus extractants were used $\left(\mathrm{D}_{2} \mathrm{EHPA}\right.$, P507 and Cyanex272). The experiments showed that, the increase in the ascorbic acid concentration enhances the extraction and selectivity of the extraction of REEs. It was also determined that the more efficient extractant in the conditions of this study was P507. We considered that the best conditions to perform the separation between La/ didymium was obtained with P507 as extraction agent P507 and concentration of ascorbic acid $0.5 \mathrm{~mol} \cdot \mathrm{L}^{-1}$. In this condition the extractions of $\mathrm{La}$, Pr and $\mathrm{Nd}$ were obtained equal to $12.3 \pm 1.3 \%, 56.4 \pm 1.0 \%, 64.8 \pm 1.7 \%$, respectively, as well as a separation factor of $9.3 \pm 0.8$ and didymium in organic phase with $81.4 \pm 0.5 \%$ purity.
\end{abstract}

KEYWORDS: Ascorbic acid; Cyanex272; D2EHPA; Rare earth elements; Liquid-liquid extraction; P507

ORCID: Gabriel Santos (https://orcid.org/0000-0001-6744-8879); Ysrael M. Vera (https://orcid.org/0000-0002-4035-2284)

Copyright: (C) 2019 CSIC. Este es un artículo de acceso abierto distribuido bajo los términos de la licencia de uso y distribución Creative Commons Reconocimiento 4.0 Internacional (CC BY 4.0). 


\section{INTRODUCCIÓN}

Los elementos tierras raras (ETRs) presentan características químicas muy similares y están constituidos por los elementos de la familia de los Lantánidos, además del itrio y el escandio. Se utilizan ampliamente en industria de alta tecnología, teniendo numerosas aplicaciones como, por ejemplo, en la fabricación de catalizadores para la industria automotriz y la petroquímica, para producir aleaciones especiales, en la producción de energía eléctrica a partir de fuentes renovables, entre otras muchas (Gupta y Krishnamurthy, 2005).

Debido a sus características químicas similares, la separación de los ETRs entre sí es una tarea compleja. La extracción líquido - líquido (ELL) es la principal técnica para separarlos, ya que es la más versátil y la que ofrece la mejor relación costo/beneficio (Ritcey, 2006).

El uso de agentes de extracción saponificados en la extracción de los ETRs es ampliamente utilizado en la industria debido a las altas extracciones y relativamente satisfactorias separaciones obtenidas (Yan et al., 2006). A pesar de estos, el uso de agentes de extracción saponificado genera soluciones acuosas conteniendo iones sodio, amonio, magnesio o calcio (depende del agente usado en la saponificación) que necesitan ser eliminadas del efluente líquido antes de su descarte o reutilización. Las técnicas más comunes usadas para la eliminación de iones del agua son la osmosis inversa, electrodiálisis, destilación o membranas de intercambio iónico (AlMarzooqi et al., 2014). El empleo de agentes acomplejantes en la ELL viene siendo estudiado, dado que esta alternativa puede proporcionar extracciones con selectividades satisfactorias y sin la generación de desechos dañinos al medio ambiente cuando el agente acomplejante es recuperado o cuando es biodegradable. Sustancias biodegradables son fáciles de tratar por medio de un proceso biológico como es el caso de lodo activado que también es más económico que las técnicas de desalinización antes mencionados (Eckenfeldey y Cleary, 2013).

Agentes acomplejantes como ácido etilendiaminotetraacético (EDTA), el ácido dietilenotriamino penta acético (DTPA), ácido láctico, ácido acético ya fueron utilizados para favorecer en la separación de ETRs usando la ELL (Nishihama et al., 2000; Wang et al., 2004; Scal et al., 2016; Gomes et al., 2017). El EDTA adicionado en una solución conteniendo $\mathrm{Ho} / \mathrm{Y} / \mathrm{Er}$ funcionó como agente enmascarante de Ho y Er dejando el Y libre para ser extraído por el agente de extracción éster mono-2-etil-hexílico do ácido 2-etil-hexil fosfónico aumentando la selectividad de la separación. La menor constante de estabilidad del complejo EDTA-Y que las constantes de los complejos formados entre el EDTA - Ho y EDTA - Er posibilitó la selectividad de la separación (Nishihama et al., 2000).
La selectividad de la separación de $\mathrm{Y}$ de una mezcla de $\mathrm{Tm} / \mathrm{Er} / \mathrm{Yb} / \mathrm{Y}$ usando el reactivo de extracción ácido s-nonilfenoxi acético (CA-100) y los agentes acomplejantes EDTA, ácido dietilenotriamino - penta acético (DTPA) aumentó y simplificó el proceso de separación de itrio debido al cambio del orden de extracción de los ETRs con el CA-100. La extracción de los elementos está influenciada por la diferencia entre las constantes de estabilidad de los ETRs con los complejos. Los valores de las constantes de estabilidad de los complejos formados entre el EDTA y los ETRs tienen el siguiente orden: $\mathrm{Y}<\mathrm{Er}<\mathrm{Tm}<\mathrm{Yb}$. Como consecuencia, la extracción del $\mathrm{Er}$ al $\mathrm{Yb}$ es más reducida que para $\mathrm{Y}$ teniendo lugar el efecto de enmascaramiento. En los sistemas estudiados todos los agentes acomplejantes forman complejos 1:1 con los ETRs y sólo los iones de ETRs libres (los no acomplejados) participan de la extracción. En el caso del EDTA, los factores de separación del $\mathrm{Y}$ con respecto al $\mathrm{Yb}, \mathrm{Tm}$ y Er aumentan con el aumento del pH (Wang et al., 2004).

En estudios anteriores realizados en el laboratorio de Hidrometalurgia del Centro de tecnología Mineral (Brasil) condicionando con ácido láctico la solución acuosa que contiene los ETRs, se ha comprobado la mejora del procedimiento de los reactivos de extracción ácidos organofosforados en la separación de didimio de lantano (Scal et al., 2016; Gomes et al., 2017). Los factores de separación entre los ETRs ligeros fueron mayores cuando el ácido láctico estaba presente en la solución de alimentación que cuando no lo estaba, en las mismas condiciones. Estos ácidos hidroxicarboxílicos son ácidos débiles que se ionizan parcialmente en agua. La base conjugada de estos ácidos actúa como un tampón de $\mathrm{pH}$ de la solución acuosa, evitando la reducción drástica del $\mathrm{pH}$ y favoreciendo la extracción y separación de ETRs. El presente trabajo tuvo como objetivo evaluar la influencia del agente de extracción y la concentración de ácido ascórbico sobre la extracción de los Elementos Tierras Raras Ligeros (La, Pr y Nd) utilizando la técnica de extracción líquido - líquido. Los agentes de extracción orgánicos utilizados fueron D2EHPA, P507 y Cyanex272.

\section{MATERIALES Y MÉTODOS}

\subsection{Reactivos químicos}

Se utilizó una solución sintética en la extracción de ETRs en los ensayos de ELL y fue preparado a partir de la solubilización de los óxidos de tierras raras en $\mathrm{HCl}$ concentrado a $300^{\circ} \mathrm{C}$. La composición de la solución sintética fue: $21,02 \mathrm{~g} \cdot \mathrm{L}^{-1} \mathrm{de}_{\mathrm{La}_{2} \mathrm{O}_{3}}$, $4,87 \mathrm{~g} \cdot \mathrm{L}^{-1}$ de $\operatorname{Pr}_{6} \mathrm{O}_{11}$ y $17,47 \mathrm{~g} \cdot \mathrm{L}^{-1}$ de $\mathrm{Nd}_{2} \mathrm{O}_{3}$. Los óxidos fueron adquiridos de la Pacific Industrial Development Corporation. La composición de la 
solución sintética es similar a la de una solución acuosa de la lixiviación de un concentrado de monazita brasileña después de separados los ETRs ligeros de los pesados (Da Luz y Lins, 2005).

La concentración de ácido ascórbico fue variada, con el objetivo de encontrar la concentración más efectiva en términos de selectividad de la extracción. Todos los ensayos de extracción se realizaron en $\mathrm{pH}$ 3,5. Las concentraciones de ácido ascórbico en fase acuosa fueron: $0 \mathrm{~mol} \cdot \mathrm{L}^{-1} ; 0,1 \mathrm{~mol} \cdot \mathrm{L}^{-1} ; 0,3 \mathrm{~mol} \cdot \mathrm{L}^{-1}$; $0,5 \mathrm{~mol} \cdot \mathrm{L}^{-1}$ y $0,7 \mathrm{~mol} \cdot \mathrm{L}^{-1}$.

Los ácidos organofosforados, ácido di-(2-etilhexil) fosfórico (D2EHPA), éster mono-2-etil-hexílico do ácido 2 - etilhexil fosfónico (P507) y el ácido bis (2,4,4 - trimetilpentil) fosfónico (Cyanex 272) se adquirieron de la Aodachem (China) y se diluyeron con isoparafina 17/21 (Ypiranga Brasil) y se obtuvieron soluciones al 20\% (v/v) (aproximadamente $0,6 \mathrm{~mol} \cdot \mathrm{L}^{-1}$ del reactivo de extracción).

\subsection{Ensayos de extracción}

Los ensayos de extracción líquido - líquido se realizaron por triplicado y a temperatura ambiente $\left(\mathrm{T}=25^{\circ} \mathrm{C}\right)$. Las desviaciones estándar de todas las variables fueron calculadas. Se utilizaron $20 \mathrm{~mL}$ de solución acuosa y $20 \mathrm{~mL}$ de solución orgánica. En los embudos de separación, se pusieron en contacto las fases y la agitación se realizó en una mesa agitadora durante $20 \mathrm{~min}$ y a $250 \mathrm{rpm}$ de velocidad de rotación. Experimentos preliminares mostraron que $5 \mathrm{~min}$ de agitación son suficientes para alcanzar el equilibrio de extracción. Después de la etapa de agitación, las fases se dejaron en reposo para su separación y posteriormente la fase acuosa fue filtrada a través de papel de filtro de celulosa por gravedad. Las soluciones acuosas se recogieron para realizar el análisis químico de los ETRs.

\subsection{Análisis químico}

Los análisis cuantitativos de praseodimio y neodimio en las soluciones acuosas y en la alimentación se realizaron con un espectrofotómetro UV-Vis (HACH 6000) en las longitudes de onda de $443 \mathrm{~nm}$ y $793 \mathrm{~nm}$, respectivamente. La concentración de ETR total se obtuvo a partir de la titulación complejométrica con ácido dietilenotriamino penta - acético (DTPA) (Pribil y Vesely, 1963). La titulación fue realizada en $\mathrm{pH} 9$, utilizando tampón de $\mathrm{NH}_{4} \mathrm{Cl} / \mathrm{NH}_{3}$, y usando el Negro de Eriocromo T (NET) como indicador. La cuantificación de lantano se realizó por diferencia entre la concentración de tierras raras total y las concentraciones de Pr y Nd.

\subsection{Ecuaciones y parámetros}

Una vez que las concentraciones de los ETRs en las soluciones acuosas y en la alimentación fueron determinadas, se calcularon los parámetros
TABLA 1. Parámetros utilizados en la evaluación de los resultados de los experimentos de extracción de ETRs por ELL

\begin{tabular}{ll}
\hline Parámetro & \multicolumn{1}{c}{ Ecuación } \\
$\begin{array}{l}\text { Porcentaje de } \\
\text { ETR extraído }\end{array}$ & $\% \mathrm{E}_{\mathrm{M}}=\frac{[\mathrm{M}]_{\mathrm{total}}-[\mathrm{M}]_{\mathrm{eq}(\mathrm{ac})}}{[\mathrm{M}]_{\mathrm{total}}} \times 100$ \\
$\begin{array}{l}\text { Coeficiente de } \\
\text { Distribución }\end{array}$ & $\mathrm{D}_{\mathrm{M}}=\frac{[\mathrm{M}]_{\mathrm{eq}(\mathrm{org})}}{[\mathrm{M}]_{\mathrm{eq}(\mathrm{ac})}}$ \\
$\begin{array}{l}\text { Factor de } \\
\text { Separación }\end{array}$ & $\beta_{\mathrm{M} 1 / \mathrm{M} 2}=\frac{\mathrm{D}_{\mathrm{M} 1}}{\mathrm{D}_{\mathrm{M} 2}}$ \\
$\begin{array}{l}\text { Pureza do } \\
\text { Didímio }\end{array}$ & $\mathrm{P}(\%)=\frac{[\mathrm{Pr}]_{\mathrm{eq}(\text { org })}+[\mathrm{Nd}]_{\mathrm{eq}(\text { org })}}{[\mathrm{Pr}]_{\mathrm{eq}(\mathrm{org})}+[\mathrm{Nd}]_{\mathrm{eq}(\mathrm{org})}+[\mathrm{La}]_{\mathrm{eq}(\mathrm{org})}} \times 100$
\end{tabular}

incluidos en la Tabla 1 para los diferentes ensayos de extracción realizados. Las ecuaciones matemáticas presentes en la Tabla 1 se utilizaron para los cálculos de estos parámetros.

En las ecuaciones de la Tabla $1[\mathrm{M}]$ total y [M] eq (ac) representan las concentraciones de un determinado ETR en la fase acuosa antes y después de la extracción, respectivamente. [M] eq (org) es la concentración del metal en la solución orgánica después de la extracción.

\section{RESULTADOS Y DISCUSIÓN}

A partir del análisis de la Fig. 1A - D es posible verificar cómo se comportó la extracción de cada ETR al utilizar los diferentes reactivos de extracción y así como la influencia de la concentración del ácido ascórbico sobre el $\mathrm{pH}$ de equilibrio.

Se percibe que los agentes de extracción orgánicos estudiados tienen mayor preferencia por el $\mathrm{Nd}$ y el Pr que por el La. Este hecho puede ser explicado por la teoría de ácidos y bases duras y blandas de Pearson. Esta teoría clasifica los ácidos y bases en duros y blandos. Las especies duras son aquellas poco polarizables, con mayor carga y menor tamaño, mientras que las blandas son polarizables, tienen menor carga y mayor tamaño. Según esta teoría los ácidos duros tienen mayor preferencia por las bases duras y los ácidos blandos tienen mayor preferencia por las bases blandas (Pearson, 1963).

Las bases conjugadas de los agentes de extracción orgánicos utilizados en los ensayos, según la teoría de Pearson, son consideradas bases duras, porque todas poseen el elemento electronegativo oxígeno. Sin embargo, sus durezas no son de la misma intensidad. Mientras que el D2EHPA es la base más dura de los tres reactivos de extracción, por poseer cuatro átomos de oxígeno ligados al fósforo, el Cyanex272 es la menos dura, pues tiene dos 

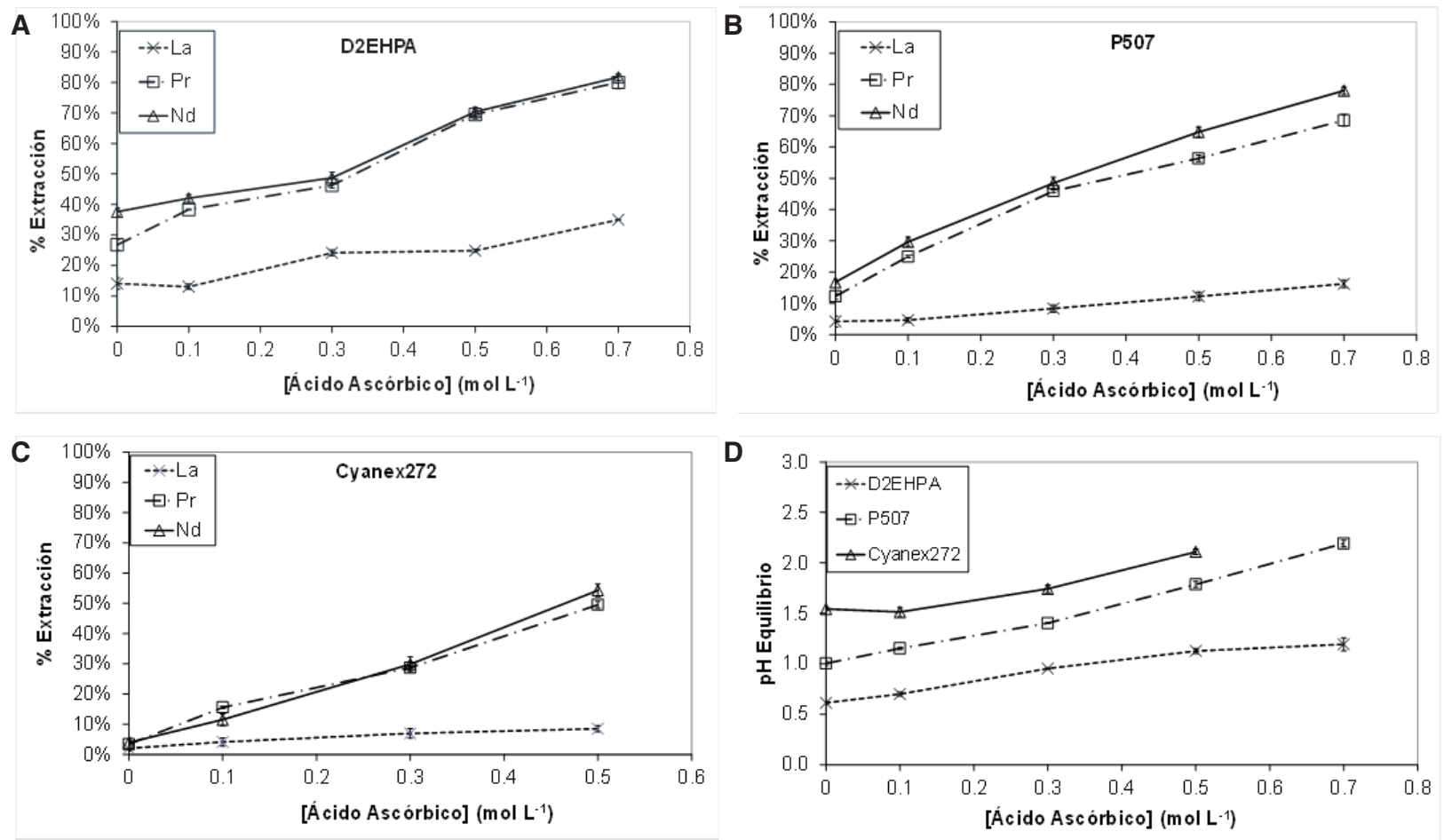

FIGURA 1. Influencia de la concentración de ácido ascórbico en la extracción de los ETRs con: (A) D2EHPA; (B) P507; (C) Cyanex272, y (D) Influencia de la concentración de ácido ascórbico en el pH de equilibrio.

átomos de oxígeno enlazados al fósforo y el P507 tiene una dureza intermedia porque tiene tres átomos de oxígeno enlazados al fósforo.

Además, los iones trivalentes de los lantánidos se consideran ácidos duros y sus radios atómicos van disminuyendo conforme el número atómico va aumentando, o sea, mientras mayor sea el número atómico del ETR, más duro será.

En los ensayos de extracción, el ETR más extraído fue el Nd por ser el ETRs más duro y tener una mayor afinidad con los agentes de extracción. El agente de extracción que obtuvo las mayores extracciones de los ETRs fue el D2EHPA, ya que es el extractor con la base conjugada más dura.

Por otra parte, se puede observar que con el aumento de la concentración de ácido ascórbico la extracción es más efectiva. Esto se debe a que el ácido ascórbico funciona como un tampón de $\mathrm{pH}$ de la fase acuosa, siguiendo las reacciones de equilibrio, Ecs. (1), (2) y (3):

$$
\begin{gathered}
\text { HAsc (ac) }=\operatorname{Asc}^{-}(\mathrm{ac})+\mathrm{H}^{+}(\mathrm{ac}) \\
\mathrm{M}^{3+}(\mathrm{ac})+3 \mathrm{H}_{2} \mathrm{~A}_{2}(\mathrm{org})=\mathrm{M}\left(\mathrm{HA}_{2}\right)_{3}(\mathrm{org})+3 \mathrm{H}^{+}(\mathrm{ac}) \\
\operatorname{Asc}^{-}(\mathrm{ac})+\mathrm{H}^{+}(\mathrm{ac})=\text { HAsc }(\mathrm{ac})
\end{gathered}
$$

El ácido ascórbico (HAsc) cuando se añade al agua se ioniza parcialmente (reacción 1). El pKa del
HAsc es 4,03 (Martell y Smith, 1977) y en pH 3,5 la ionización es de $22,8 \%$. En la reacción (2), se observa el equilibrio de extracción entre el solvente $\left(\mathrm{H}_{2} \mathrm{~A}_{2}\right)$ y el ETR $\left(\mathrm{M}^{3+}\right)$. Al agregar ácido ascórbico, el mismo impide que el $\mathrm{pH}$ del medio disminuya drásticamente, pues los protones liberados en la extracción son consumidos por el ion ascorbato ( $\mathrm{Asc}^{-}$) generado en el proceso de ionización de la molécula de ácido ascórbico, como se puede observar en la reacción (3). El ion ascorbato tiene que estar presente en solución para que se forme el sistema tampón de $\mathrm{pH}$ en solución (ácido ascórbico/ascorbato). El grado de ionización del ácido débil aumenta cuando aumenta el $\mathrm{pH}$ de la solución, siendo que el grado de ionización es $50 \%$ cuando el $\mathrm{pH}$ de la solución es numéricamente igual al pKa del ácido débil, que en este caso es 4,03. Por otro lado, el aumento del $\mathrm{pH}$ de la solución provoca la formación y precipitación de los hidróxidos de ETRs lo que limita el aumento del $\mathrm{pH}$ de trabajo. La precipitación de los hidróxidos de ETRs en la solución acuosa de extracción comenzó aproximadamente a partir del $\mathrm{pH} 4,0$ y por estas razones es que los ensayos de extracción fueron realizados en $\mathrm{pH}$ 3,5.

Al analizar las ecuaciones 1, 2 y 3 es posible percibir que la reacción de extracción es favorecida con el aumento de la concentración del ácido ascórbico porque más iones ascorbato serán producidos y más iones hidrógeno podrán ser consumidos $\mathrm{y}$, consecuentemente, el equilibrio de la reacción 

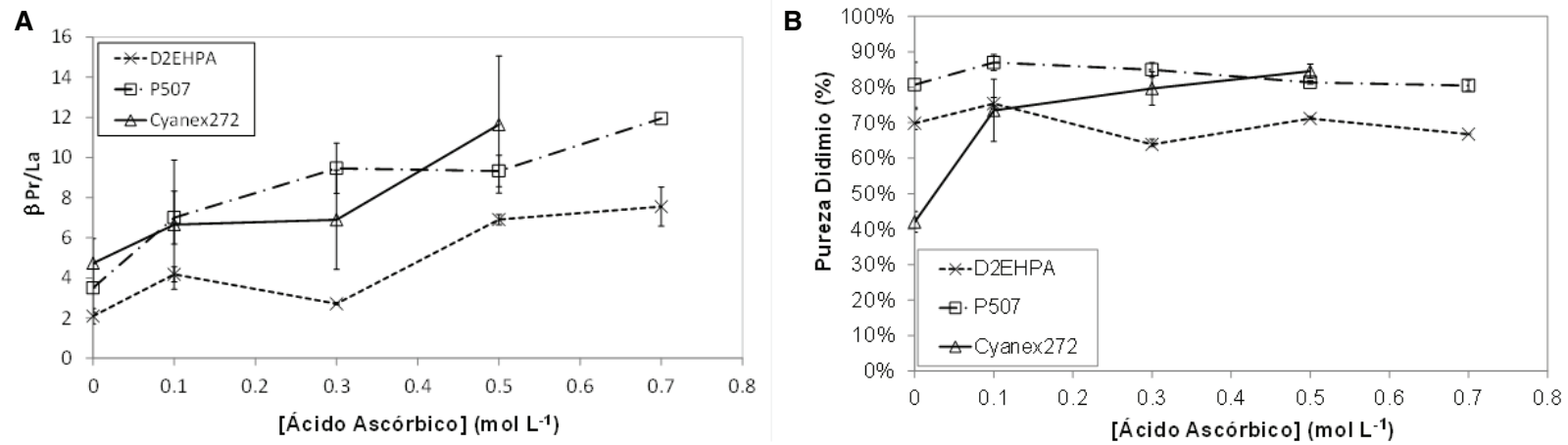

Figura 2. (A) Factor de separación Pr/La; y (B) Pureza de Didimio en función del agente de extracción y de la concentración de HAsc.

de extracción se desplazará más hacia la formación de los productos. De este modo, al aumentar la concentración del ácido ascórbico, se produce un aumento del $\mathrm{pH}$ de equilibrio, pues más protones son consumidos por los iones ascorbato (Fig. 1D).

El ácido ascórbico a diferencia de EDTA y DTPA no funciona como agente acomplejante de los ETRs porque la reacción de acomplejación entre los ETRs y la base conjugada (ascorbato) ocurre en poca extensión (valor máximo calculado fue de $2 \%$ ) porque las constantes de estabilidad de los complejos ETRs Asc son relativamente pequeñas $\left(\log \beta_{\mathrm{La}}=1,41\right.$; $\log \beta_{\mathrm{Pr}}=1,48$ e $\log \beta_{\mathrm{Nd}}=1,54$, Fuerza iónica igual a $\left.2,0 \mathrm{~mol} \cdot \mathrm{L}^{-1}\right)$, además, porque solamente ocurre la adición de una molécula de ascorbato al ETR se formando un complejo con carga aun positiva que es extraído por el reactivo de extracción catiónico (Ec. 4), (Hasegawa et al., 1988):

$$
\mathrm{M}^{3+}(\mathrm{ac})+\mathrm{Asc}^{-}(\mathrm{ac})=\mathrm{MAsc}^{2+}(\mathrm{ac})
$$

La Fig. 2 (A-B) muestran la variación del factor de separación $\mathrm{Pr} / \mathrm{La}$ y la pureza del didimio en la fase orgánica cargada en función de la concentración de ácido ascórbico para cada agente de extracción estudiado.

El parámetro $\beta \mathrm{Pr} / \mathrm{La}$ se refiere a la selectividad de la extracción del Pr/La. Al observar la Fig. 2A, es posible verificar que la adición del ácido ascórbico aumenta la selectividad de la extracción Pr/La para los tres agentes de extracción. Los agentes de extracción más selectivos fueron el P507 y el Cyanex272. Sin embargo, el agente de extracción P507 produce mejores extracciones que el Cyanex272. En el caso del D2EHPA, se verificó lo contrario, hubo una menor selectividad y un mayor porcentaje de extracción.

La precipitación, en la fase orgánica, del complejo formado entre el agente de extracción y el ETR es otro aspecto importante para ser tenido en consideración a la hora de escoger la mejor condición de extracción. La precipitación del complejo imposibilita la operación de extracción líquido - líquido en las condiciones operacionales en que aparece el precipitado. La precipitación del complejo depende de la solubilidad de este en fase orgánica y de la concentración del complejo formado. En el caso del D2EHPA la extracción de los ETRs es muy alta por lo que a partir de $0,3 \mathrm{~mol} \cdot \mathrm{L}^{-1}$ de HAsc tiene lugar la precipitación del complejo. En el caso do P507 la precipitación del complejo en fase orgánica se produjo apenas en 0,7 $\mathrm{mol} \cdot \mathrm{L}^{-1}$ de HAsc y en el caso de Cyanex272 a partir de concentraciones de HAsc igual a $0,3 \mathrm{~mol} \cdot \mathrm{L}^{-1}$ siendo que en la condición de $0,7 \mathrm{~mol} \cdot \mathrm{L}^{-1}$ no fue posible retirar muestra de fase acuosa después de la extracción para analizar el contenido de ETRs. El complejo formado entre el Cyanex272 y los ETRs tienen menor solubilidad en fase orgánica lo que queda evidenciado porque a pesar de que el Cyanex 272 presenta las menores extracciones de ETRs, se observó la aparición de precipitado durante la extracción.

La Fig. 2B muestra la variación de la pureza de didímio en función de la concentración de ácido ascórbico para cada solvente. Con el D2EHPA se obtuvieron las purezas más bajas, ya con el Cyanex272 (70-85\%) y el P507 (80-87\%) se obtuvieron los mejores resultados en ese aspecto.

Considerando los resultados aquí analizados (porcentaje de extracción, factor de separación Pr/ La y precipitación de complejo en fase orgánica) consideramos que la mejor condición para realizar la separación entre La/didímio es con el solvente P507 y concentración de HAsc en la solución acuosa igual a $0,5 \mathrm{~mol} \cdot \mathrm{L}^{-1}$. En esta condición se obtuvo una extracción de La, Pr y Nd igual a $12,3 \pm 1,3 \%, 56,4$ $\pm 1,0 \%, 64,8 \pm 1,7 \%$, respectivamente, así como un factor de separación de 9,3 $\pm 0,8$ y didímio en fase orgánica con $81,4 \pm 0,5 \%$ de pureza.

\section{CONCLUSIONES}

- En este trabajo se evaluó la influencia de la adición de ácido ascórbico sobre la extracción de didimio y lantano, a partir de la extracción líquido - líquido, empleando ácidos 
organofosforados como reactivos de extracción. En los ensayos se emplearon como agentes de extracción D2EHPA, P507 y Cyanex272, y se varió la concentración en fase acuosa del ácido ascórbico.

- Se verificó que el aumento de la concentración de ácido ascórbico mejora el porcentaje de extracción y la selectividad de la extracción de los ETR.

- Como conclusión principal se puede decir que, entre los reactivos de extracción utilizados, el P507 es considerado el más adecuado para trabajar en conjunto con el ácido ascórbico en las condiciones estudiadas y la utilización del ácido ascórbico es adecuado para ser una alternativa a la saponificación de los ácidos organofosforados. Los trabajos futuros de este estudio contemplan la determinación de los parámetros de operación de la extracción líquido - líquido en régimen continuo: número de etapas de extracción, y la relación entre el caudal volumétrico del solvente orgánico y de la solución de alimentación. Además, se realizará el estudio de la eliminación del lantano presente en la fase orgánica cargada de ETRs (etapa de lavado). Por último, se estudiará la etapa de reextracción.

\section{AGRADECIMIENTOS}

Los autores agradecen al Consejo Nacional de Desarrollo Científico y Tecnológico $(\mathrm{CNPq})$ por la concesión de la beca a Gabriel Santos y al Centro de Tecnología Mineral de Brasil por la disponibilidad de las instalaciones y todo el material necesario para el desarrollo de este estudio.

\section{REFERENCIAS}

AlMarzooqi, F.A., Al Ghaferi, A.A., Saadat, I., Hilal, N. (2014). Application of Capacitive Deionisation in water desalination: A review. Desalination 342, 3-15. https://doi. org/10.1016/j.desal.2014.02.031.

Da Luz, A.B., Lins, F.F. (2005). Rochas \& Minerais Industriais. Usos e Especificacões. Terras Raras. CETEM/MCT, Rio de Janeiro, pp. 629-654.

Eckenfelder, W.W. Jr, Cleary, J.G. (2013). Activated Sludge Technologies for Treating Industrial Wastewaters: Design and Troubleshooting. DEStech Publications Inc., Lancaster, Pennsylvania.

Gomes, R.C., Seruff, L.A, Scal, M.L.W., Vera, Y.M. (2017). The influence of lactic acid concentration on the separation of light rare earth elements by continuous liquid-liquid extraction with 2-ethylhexyl phosphonic acid mono2-ethylhexyl ester. Metal. Mater. Trans. B 49 (1), 460-465. https://doi.org/10.1007/s11663-017-1124-4.

Gupta, C.K., Krishnamurthy, N. (2005). Extractive Metallurgy of Rare Earth. CRC Press, Boca Raton, Florida.

Hasegawa, Y., Sugawara, T., Choppin, G.R. (1988). Thermodynamic parameters of complexation of lanthanoid(II1) with ascorbic acid. Inorg. Chim. Acta 143 (2), 277-280. https:// doi.org/10.1016/S0020-1693(00)83701-9.

Martell, A.E., Smith. R.M. (1977). Critical Stability Constants, Other ligands. Vol. 3, Plenum Press, New York.

Nishihama S., Hirai T., Komaswa, I. (2000). Selective Extraction of $\mathrm{Y}$ from a Ho/Y/Er Mixture by Liquid-Liquid Extraction in the Presence of a Water-Soluble Complexing Agent. Ind. Eng. Chem. Res. 39 (10), 3907-3911. https:// doi.org/10.1021/ie000030a.

Pearson, R.G. (1963). Hard and soft acids and bases. J. Am. Chem. Soc. 85 (22), 3533-3539. https://doi.org/10.1021/ja00905a001.

Pribil, R., Vesely, V. (1963). Contributions to the basic problems of Complexometry-XII. Successive determination of thorium, rare earths and some other elements. Talanta 10 (8), 899-903. https://doi.org/10.1016/0039-9140(63)80251-9.

Ritcey, G.M. (2006). Solvent Extraction. Principles and Applications to Process Metallurgy. 2nd Edition, G.M. Ritcey \& Associates Incorporated, Ottawa.

Scal, M.L.W., Seruff, L.A., Vera, Y.M. (2016). Didymium separation from lanthanum by solvent extraction using 2-ethylhexylphosphonic acid mono-2-ethylhexyl ester and lactic acid. Tecnol. Metal. Mater. Mine. 13, 373-380. https://doi. org/10.4322/2176-1523.1131

Wang, Y.G., Xiong, Y., Meng, S.L., Li, D.Q. (2004). Separation of yttrium from heavy lanthanide by CA-100 using the complexing agent. Talanta 63 (2), 239-243. https://doi. org/10.1016/j.talanta.2003.09.034.

Yan, C., Jia, J., Liao, C., Wu, S., Xu, G. (2006). Rare Earth Separation in China. Tsinghua Sci. Technol. 11 (2), 241-247. https://doi.org/10.1016/S1007-0214(06)70183-3. 